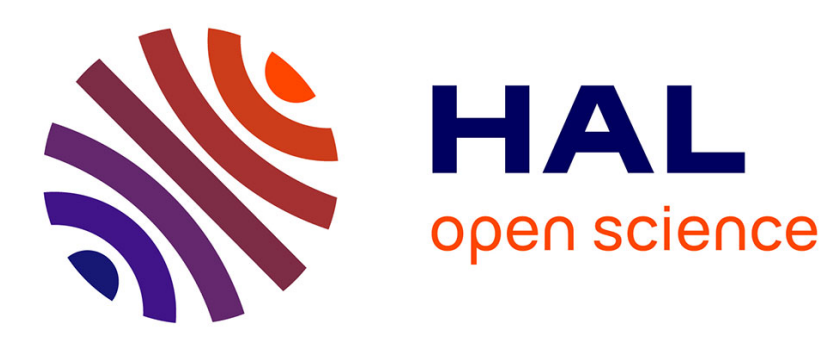

\title{
Electron-vibration energy exchange models in nitrogen plasma flows
}

\author{
A. Bourdon, P. Vervisch
}

\section{To cite this version:}

A. Bourdon, P. Vervisch. Electron-vibration energy exchange models in nitrogen plasma flows. Physical Review E , 1997, 55 (4), pp.4634-4641. 10.1103/PhysRevE.55.4634 . hal-02461787

\section{HAL Id: hal-02461787 \\ https://hal.science/hal-02461787}

Submitted on 14 Jul 2020

HAL is a multi-disciplinary open access archive for the deposit and dissemination of scientific research documents, whether they are published or not. The documents may come from teaching and research institutions in France or abroad, or from public or private research centers.
L'archive ouverte pluridisciplinaire HAL, est destinée au dépôt et à la diffusion de documents scientifiques de niveau recherche, publiés ou non, émanant des établissements d'enseignement et de recherche français ou étrangers, des laboratoires publics ou privés. 


\title{
Electron-vibration energy exchange models in nitrogen plasma flows
}

\author{
A. Bourdon* and P. Vervisch \\ URA CNRS 230 CORIA, Université de Rouen, 76821 Mont Saint Aignan Cedex, France
}

(Received 27 June 1996)

\begin{abstract}
This work presents an examination of the validity of the simple linear Landau-Teller-type model proposed by Lee for the electron-vibration energy exchange term in nitrogen [in Thermal Design of Aeroassisted Orbital Transfer Vehicles, edited by H. F. Nelson (AIAA, New York, 1985), Vol. 96, p. 3; in Thermophysical Aspects of Re-entry Flows, edited by J. N. Moss and C. D. Scott (AIAA, New York, 1986), Vol. 103, p. 197]. Plasma flow conditions encountered in high enthalpy wind tunnels are considered. The time-dependent relaxation of the vibrational energy of nitrogen due to electron inelastic collisions is calculated. The influence of the anharmonicity of the molecule and of the initial vibrational temperature $T_{v}$ is studied. With a harmonic oscillator approximation, it is found that a linear Landau-Teller-type model is accurate to describe the vibrational energy relaxation rate for electron temperatures $T_{e}$ in the range $3000 \mathrm{~K} \leqslant T_{e} \leqslant 20000 \mathrm{~K}$. When $T_{v}$ $<T_{e}$, our results show that the initial model proposed by Lee overestimates by a mean factor of 3 the electron-vibration $(e-V)$ relaxation time. When $T_{v}>T_{e}$, the relaxation time appears to depend on the initial vibrational distribution. When the anharmonicity of nitrogen is taken into account, generally the relaxation rate of the vibrational energy deviates from a linear rate equation. In this case, it appears much more difficult to model accurately the $e-V$ energy exchange term. [S1063-651X(97)02904-8]
\end{abstract}

PACS number(s): 52.30.- q

\section{INTRODUCTION}

The accurate modeling of high velocity plasma flows as encountered during the reentry of spatial vehicles requires the validation of a large number of physical and chemical models. Numerous validation studies have been carried out in plasma flows generated in high enthalpy wind tunnels where experimental data are available. However, significant discrepancies remain between measurements and computation results. Currently, different authors [1,2] suggest that a more accurate modeling of the vibrational energy relaxation in the flow may improve significantly the agreement between experiments and computations. Indeed, the vibrational energy relaxation has a significant influence on the flow since it affects not only the translational temperature and the electron temperature [through, respectively, the translation-vibration $(T-V)$ and the electron-vibration $(e-V)$ energy exchange processes] but also the chemical reactions in the flow. For the $T$ - $V$ energy exchange term, two models are very popular. The first one is the linear Landau-Teller equation [3] and the other model, proposed by Park [4], attempts to simulate the diffusive nature of vibrational relaxation. Lately, new models have been proposed to take into account the coupling between the vibrational relaxation and dissociation processes [5]. On the $e-V$ energy exchange term, the main work is the theoretical one carried out by Lee [6,7]. This author studied the vibrational relaxation of a system of molecules (considered as harmonic oscillators) populated in the ground state at $t=0 \mathrm{~s}$ which interchange energy with an electron heat bath having a constant temperature $T_{e}$. Using a theoretical approach similar to the one developed for the $T-V$ diffusion model $[4,8]$, Lee derived an analytical expression of the vibrational energy relaxation rate due to $e-V$ processes. Then,

*Electronic address: Anne.Bourdon@coria.fr studying the feature of his analytical result, Lee proposed to describe approximately the vibrational energy relaxation rate by a Linear Landau-Teller-type rate equation of the form [6]

$$
\frac{d E_{v}}{d t}=\frac{E_{v}^{*}-E_{v}}{\tau_{e}}
$$

where $E_{v}$ is the vibrational energy per unit volume, $E_{v}^{*}$ is the equilibrium energy at the electron temperature $T_{e}$, and $\tau_{e}$ is the $e-V$ relaxation time. This very simple model is generally implemented in plasma flow codes whatever the vibrational excitation of the studied flow. It is interesting to point out that $e-V$ energy exchange processes are very efficient in $\mathrm{N}_{2}$ in comparison to other molecules such as $\mathrm{O}_{2}$ or NO. Then, in the computation of plasma flows containing $\mathrm{N}_{2}$ molecules $[9,10]$, the $e-V$ energy exchange term is generally simply written as

$$
Q_{e-V}=\rho_{\mathrm{N}_{2}} \frac{e_{v, \mathrm{~N}_{2}}^{*}-e_{v, \mathrm{~N}_{2}}}{\tau_{e}}
$$

in $\mathrm{W} \mathrm{m}{ }^{-3}$, where $\rho_{\mathrm{N}_{2}}$ is the local density of $\mathrm{N}_{2}$ in the medium, $e_{v, \mathrm{~N}_{2}}$ is the local vibrational energy of $\mathrm{N}_{2}$ per unit mass, $e_{v, \mathrm{~N}_{2}}^{*}$ is the vibrational energy which corresponds to the equilibrium at the electron temperature $T_{e}$, and $\tau_{e}$ is the $e-V$ relaxation time in $\mathrm{N}_{2}$. With this simple model, the $e-V$ energy exchange appears to be much more efficient than the $T-V$ one in numerous nitrogen plasma flow conditions and the local vibrational temperature of $\mathrm{N}_{2}$ is required to be equal to the local electron temperature [10].

In this paper we question the accuracy of the simple Landau-Teller-type model for the $Q_{e-V}$ term in the computa- 
TABLE I. Constants in the $e-V$ excitation rate coefficients $k_{0, \nu}^{e-V}\left(T_{e}\right)$ for the $0 \rightarrow \nu$ transitions for $3000 \mathrm{~K} \leqslant T_{e} \leqslant 20000 \mathrm{~K}$.

\begin{tabular}{rlcccc}
\hline \hline$\nu$ & \multicolumn{1}{c}{$a$} & $b$ & $c$ & $d$ & $f$ \\
\hline 1 & -2.8583 & 8.5312 & 57.845 & -11.224 & -5.9229 \\
2 & -2.0860 & 9.0375 & 30.029 & -3.2660 & -2.9710 \\
3 & -1.6909 & 8.3733 & 16.104 & -2.2943 & 2.1450 \\
4 & -1.0249 & 3.7722 & 11.157 & -1.2874 & 2.1450 \\
5 & -0.8552 & 4.1914 & 6.1267 & -0.7564 & 2.5188 \\
6 & -0.6079 & 3.2742 & 3.5527 & -0.3861 & 2.6900 \\
7 & -0.2871 & 1.5159 & 1.8277 & -0.1870 & 2.8400 \\
8 & -0.1304 & 0.6479 & 0.8857 & -0.0485 & 3.1300 \\
9 & -0.052 & 0.3169 & 0.2218 & -0.0188 & 3.3620 \\
\hline \hline
\end{tabular}

tion of nonequilibrium plasma flows containing $\mathrm{N}_{2}$ molecules. We focus on supersonic or hypersonic plasma flows encountered in wind tunnels where the measured electron temperature may be up to about $20000 \mathrm{~K}$ in the flow [9-11] and down to $2000 \mathrm{~K}$ at the vicinity of blunt bodies [12]. The characteristic vibrational temperature of these flows is generally much more difficult to measure but remains between 2000 and $20000 \mathrm{~K}$. We propose a study of the $Q_{e-V}$ term based on the numerical solution of the master equations for the vibrational level populations of $\mathrm{N}_{2}$. First, we compare our results with those initially obtained by Lee when all the molecules are initially in their ground state. Then, we discuss the influence of the vibrational excitation of the $\mathrm{N}_{2}$ molecules on the modeling of the $Q_{e-V}$ term. In each case, the influence of the anharmonicity of $\mathrm{N}_{2}$ is studied.

\section{METHOD OF CALCULATION}

\section{A. Vibrational model for $\mathbf{N}_{2}$}

With a harmonic oscillator approximation, 34 vibrational levels lie under the dissociation limit. However, this approximation is too rough for nitrogen and we have followed the approach proposed by Dmitrieva and co-workers $[13,14]$, who considered a N-N potential which has the correct asymptotic behavior when the interatomic distance increases. In this case, 65 excited vibrational levels lie under the dissociation limit.

\section{B. Vibrational kinetics}

We neglect the influence of dissociation and recombination processes and consider only vibrational excitation and deexcitation processes by electron impact:

$$
\mathrm{N}_{2}(w)+e \leftrightarrow \mathrm{N}_{2}(v>w)+e .
$$

For an electron incident energy lower than $4 \mathrm{eV}$, the $e-\mathrm{N}_{2}$ vibrational excitation process is dominated by the well known $2.3 \mathrm{eV}^{2} \Pi_{g}$ resonance. That is, the incident electron is captured in the neighborhood of the target $\mathrm{N}_{2}$ molecule, thus forming a temporary $\mathrm{N}_{2}{ }^{-}$ion $[15,16]$. This resonance has been studied extensively theoretically and experimentally for the $0 \rightarrow v$ transitions [17-19]. The associated cross sections are dominated by a series of narrow peaks separated by just a few tenths of an electron volt which correspond to the positions of the $\mathrm{N}_{2}{ }^{-}$vibrational states. On the whole, theo- retical values agree fairly well with experimental data. For the present work, we have used the experimental $0 \rightarrow v$ transition cross sections derived by Allan [19] up to $v=13$. The corresponding $e-V$ excitation rate coefficients $k_{0, v}^{e-V}\left(T_{e}\right)$ have been obtained by numerical integration of the cross sections over a Maxwellian distribution function for the electrons. These rate coefficients can be fitted to the simple expression

$$
k_{0, v}^{e-V}\left(T_{e}\right)=10^{-15}\left(a / x+b+c x+d x^{2}\right) x^{-3 / 2} \exp (-f / x)
$$

in $\mathrm{m}^{3} \mathrm{~s}^{-1}$, where $x=T_{e}(K) / 10000 \mathrm{~K}$ and the constants are given in Table I for $1 \leqslant v \leqslant 10$ and $3000 \mathrm{~K} \leqslant T_{e} \leqslant 20000 \mathrm{~K}$. These rate coefficient values decrease with $v$ approximately as $k_{0, v}^{e-V}\left(T_{e}\right) \approx 2 k_{0, v+1}^{e-V}\left(T_{e}\right)$. The associated deexcitation rates $k_{v, 0}^{e-V}\left(T_{e}\right)$ have been derived using the detailed balance relationships. Due to the lack of accurate experimental information on excitation cross sections from a vibrational level $w \neq 0$, Capitelli and Dilonardo [20] have assumed that rate coefficients of the $w \neq 0 \rightarrow v>w$ transitions are equal to those of the $0 \rightarrow v-w$ transitions. However, since the resonant excitation process exists only for low-lying levels, different authors $[16,21]$ have shown that the excitation cross sections calculated for a few $w \neq 0 \rightarrow v>w$ transitions decrease as $w$ increases. For the purpose of the present study, the cross sections for all transitions are required. Then we have used an analytical expression of the $e-V$ cross sections between excited levels [22]:

$$
\sigma(v \leftrightarrow v+n)=\sigma(0 \leftrightarrow n)(1+0.05 v)^{-1},
$$

which is in fairly good agreement with current theoretical results $[16,21]$.

\section{Master equations}

The studied system is a collection of $\mathrm{N}_{2}$ molecules which start at $t=0 \mathrm{~s}$ to interchange energy with an electron heat bath having a constant temperature $T_{e}$. Due to $e-V$ processes, the rate of change of the number density of a vibrational level $v$ of $\mathrm{N}_{2}$, denoted $N_{v}\left(\mathrm{~m}^{-3}\right)$, is given by

$$
\frac{d N_{v}}{d t}=n_{e} \sum_{\substack{w=0 \\ w \neq v}}^{v_{\max }}\left[k_{w, v}^{e-V}\left(T_{e}\right) N_{w}-k_{v, w}^{e-V}\left(T_{e}\right) N_{v}\right],
$$




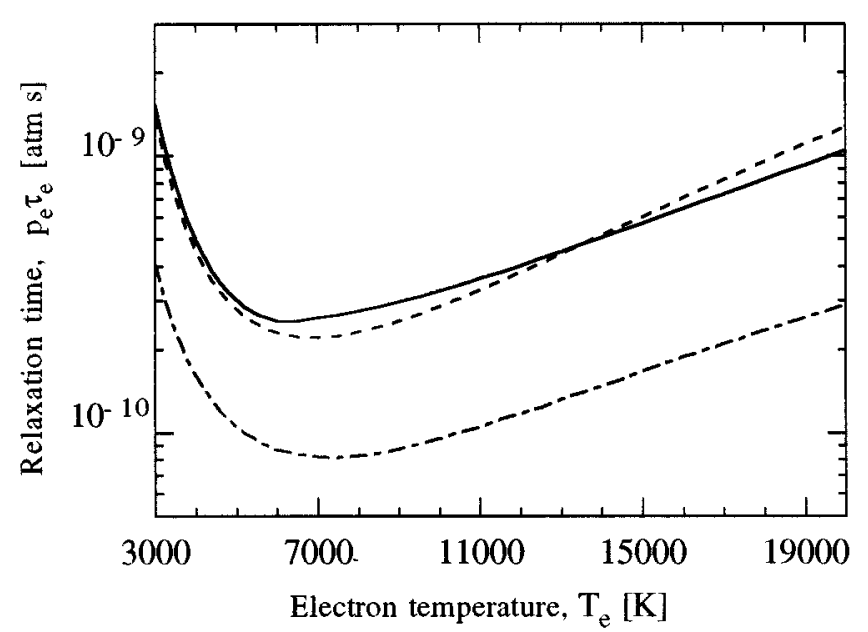

FIG. 1. Comparison of $e-V$ relaxation times. Solid line, $p_{e} \tau_{e \text { Lee }}[7,9]$; dashed line, Eq. (8) with the cross sections derived by Allan [19]; dot-dashed line, $p_{e} \tau_{e c}$.

where $n_{e}\left(\mathrm{~m}^{-3}\right)$ is the electron number density and $v_{\max }$ is the number of excited vibrational levels $\left(v_{\max }=33\right.$ in the harmonic case and $v_{\max }=65$ in the anharmonic case). We note $N_{t}=\Sigma N_{v}$ the total $\mathrm{N}_{2}$ number density. The system of differential equations has been integrated numerically in using LSODE [23], a routine specifically designed to solve stiff ordinary differential equations. In each set of calculations, we keep $T_{e}, n_{e}$, and $N_{t}$ constant. Considering the energy of the level $w=0$ as reference, the vibrational energy of the studied system is given by

$$
E_{v}=\sum_{w=1}^{v_{\max }} N_{w} \epsilon_{w}
$$

where $\epsilon_{w}$ is the energy of the vibrational level $w$. The numerical approach used in this paper allows us to follow the temporal relaxation of $d E_{v} / d t, E_{v}$, and of vibrational level populations.

\section{STUDY OF THE VALIDITY OF THE MODEL PROPOSED BY LEE}

\section{A. Analytical results proposed by Lee}

To implement the simple model proposed by Lee in flow codes [Eq. (2)], the only need is to determine the $e$ - $V$ relaxation time. Lee proposed an analytical expression of the form

$$
\tau_{e \text { Lee }}=\left\{\frac{n_{e}}{2}\left[1-\exp \left(\frac{-\theta_{v}}{T_{e}}\right)\right]^{2} \int k_{0, v}^{e-V} v^{2} d v\right\}^{-1}
$$

in $s$, where $\theta_{v}$ is the characteristic vibrational temperature of the molecule. This relaxation time is independent of the molecular number density but depends on $n_{e}$ and $T_{e}$. Using Slinker and Ali cross sections [24], Lee [7] calculated the $e-V$ relaxation time in $\mathrm{N}_{2}$. As the lattter depends on $n_{e}$, Candler [9] has curve fit $p_{e} \tau_{e \text { Lee }}$ where $p_{e}$ is the electron partial pressure in atmospheres. Results are given in Fig. 1 for $3000 \mathrm{~K} \leqslant T_{e} \leqslant 20000 \mathrm{~K}$. We note that the relaxation time is the smallest at about $T_{e}=7000 \mathrm{~K}$ and increases rapidly on either side of this electron temperature. The very small re-

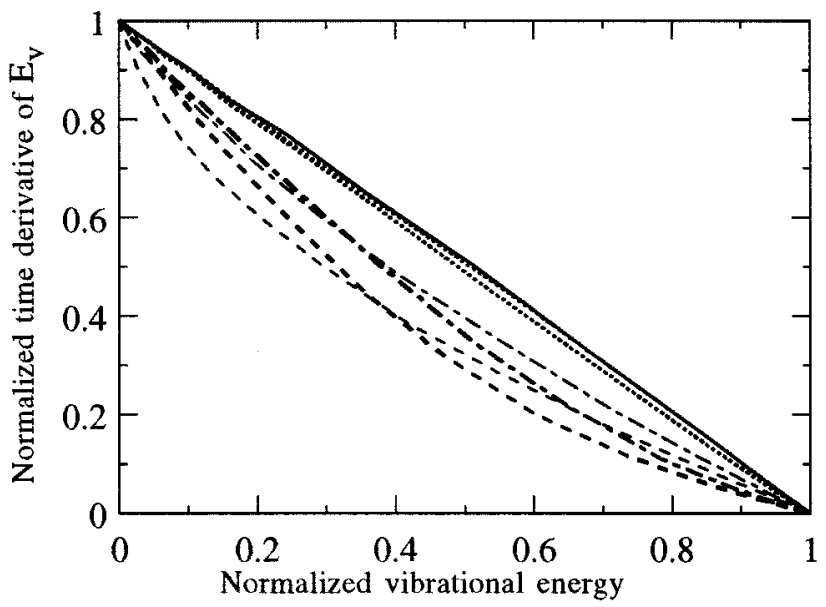

FIG. 2. With a harmonic oscillator approximation, the influence of $T_{e}$ on the deviations from a Landau-Teller-type rate equation for the initial condition studied by Lee. Thin dashed line, $T_{e}=2000 \mathrm{~K}$; thin dot-dashed line, $T_{e}=3000 \mathrm{~K}$; thin dotted line, $T_{e}=6000 \mathrm{~K}$; thin solid line, $T_{e}=8000 \mathrm{~K}$; thick dotted line, $T_{e}=10000 \mathrm{~K}$; thick dotdashed line, $T_{e}=20000 \mathrm{~K}$; thick dashed line, $T_{e}=30000 \mathrm{~K}$.

laxation time obtained near $7000 \mathrm{~K}$ may be attributed to the strong resonance between the electron and vibrational modes. In Fig. 1, we note that the relaxation time calculated with Eq. (8) and more accurate experimental cross sections [19] remains very close to the one initially proposed by Lee.

\section{B. Numerical study of the $e-V$ energy exchange term}

We consider the same initial condition as the one studied by Lee: at $t=0 \mathrm{~s}$, all $\mathrm{N}_{2}$ molecules are populated in the ground state. This situation may represent a plasma flow in the vicinity of a shock wave around a blunt body. In this case, the vibrational energy is zero at $t=0 \mathrm{~s}$ and increases with time up to the value $E_{v}^{*}$ which corresponds to the vibrational energy of the system of molecules in Boltzmann equilibrium at $T_{e}$. On the contrary $d E_{v} / d t$ is maximum at $t=0 \mathrm{~s}$ and decreases down to zero at the final equilibrium. If the relaxation of the vibrational energy follows a LandauTeller-type relaxation, the curve $d E_{v} / d t=f\left(E_{v}\right)$ is a straight line and its slope is inversely proportional to the relaxation time $\tau_{e}$.

\section{1. $\mathrm{N}_{2}$ is considered as a harmonic oscillator}

Figure 2 presents the time evolution of $d E_{v} / d t$ as a function of $E_{v}$ for different electron temperatures. We normalized $E_{v}$ by the equilibrium energy $E_{v}^{*}$ and $d E_{v} / d t$ by its value at $t=0 \mathrm{~s}$, then the results of Fig. 2 are independent of $n_{e}$ and $N_{t}$ and depend only on $T_{e}$. We note that the computed vibrational energy relaxation rate can be considered to be linear with a relative discrepancy lower than $30 \%$ for $3000 \mathrm{~K} \leqslant T_{e} \leqslant 20000 \mathrm{~K}$. In fact, the deviations observed for low electron temperatures were expected since the vibrational excitation of $\mathrm{N}_{2}$ occurs only when the incident electrons are energetic enough. But, Fig. 2 also shows that a linear model is inaccurate at high electron temperatures. This confirms that the linear model is only an approximation of 


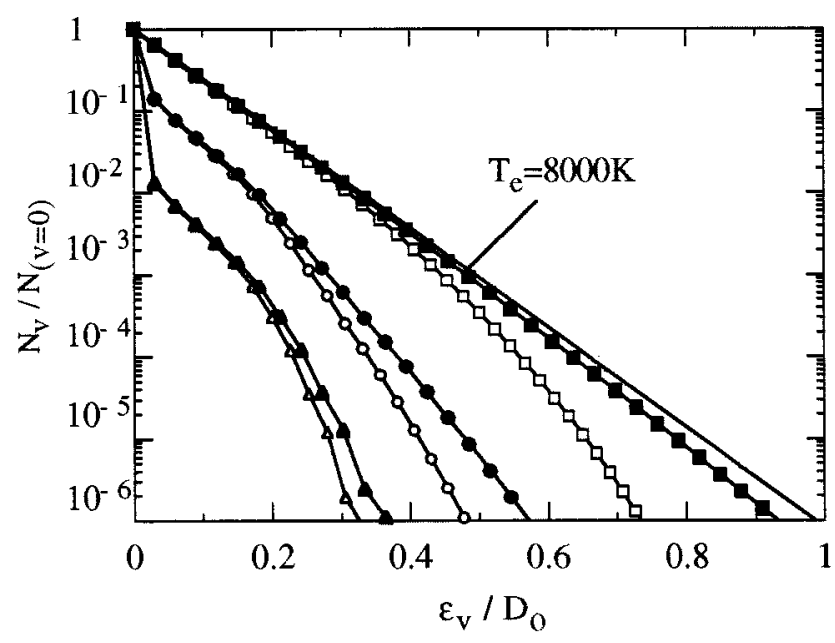

FIG. 3. Temporal relaxation of the vibrational level populations for $T_{e}=8000 \mathrm{~K}$ with, at $t=0 \mathrm{~s}$, all the $\mathrm{N}_{2}$ molecules populated in the ground state. The number density $N_{v}$ is normalized by the number density on the ground state and $\epsilon_{v}$ by the dissociation energy $D_{0}$ of $\mathrm{N}_{2}$. The time $t$ is normalized by $\tau_{e \text { Lee }}$ calculated at $T_{e}=8000 \mathrm{~K}$. Closed symbols correspond to the harmonic oscillator approximation; open symbols correspond to the anharmonic oscillator approximation. Triangles, $t=10^{-2} \tau_{e \text { Lee }}$; circles, $t=10^{-1} \tau_{e \text { Lee }}$; squares, $t=\tau_{e \text { Lee }}$. The solid line corresponds to the final Boltzmann distribution at $T_{e}$. This equilibrium is reached at $t=7.2 \tau_{e \text { Lee }}$ in the harmonic case and at $t=1363 \tau_{e \text { Lee }}$ in the anharmonic case.

the analytical solution derived by Lee with a classical approximation (valid at high electron temperatures.) In fact, Fig. 2 seems to indicate that the simple linear model is valid only in the temperature range where the $e-V$ relaxation time derived by Lee is the smallest $\left(T_{e} \approx 7000 \mathrm{~K}\right)$. For example, for $T_{e}=8000 \mathrm{~K}$, Fig. 3 shows the time evolution of the population $N_{v}$ of each vibrational level as a function of its vibrational energy $\epsilon_{v}$. We normalize $N_{v}$ by the number density on the ground state and $\epsilon_{v}$ by the dissociation energy $D_{0}$ of $\mathrm{N}_{2}$. When the vibrational levels are populated according to a Boltzmann distribution, the slope of the distribution is linear and is inversely proportional to the vibrational temperature $T_{v}$. We have also normalized the time by $\tau_{e \text { Lee }}$ calculated at $T_{e}=8000 \mathrm{~K}$, then the results obtained in Fig. 3 are independent of $n_{e}$ and $N_{t}$ and depend only on $T_{e}$. As expected, we note that lower levels relax quicker than higher ones. During the relaxation, the vibrational distribution remains different from a Boltzmann distribution, even for low vibrational levels. This result points out that the linear Landau-Teller-type relaxation rate observed in Fig. 2 for $T_{e}=8000 \mathrm{~K}$ is not the result of the relaxation of a particular vibrational distribution.

Finally, for $3000 \mathrm{~K} \leqslant T_{e} \leqslant 20000 \mathrm{~K}$, we have linearized our computed $d E_{v} / d t=f\left(E_{v}\right)$ curves and we have used the slopes to derive new $e-V$ relaxation time values as a function of $T_{e}$. Figure 1 compares this $e-V$ relaxation time (denoted $\tau_{e c}$ ) with the one initially proposed by Lee (denoted $\tau_{e \text { Lee }}$ ). We note that $\tau_{e c}$ varies as a function of $T_{e}$ in the same way as $\tau_{e \text { Lee }}$ but $\tau_{e c}$ remains smaller than $\tau_{e \text { Lee }}$ in the whole temperature range. The smallest discrepancy is about a factor of 2.5 at $T_{e}=5000 \mathrm{~K}$ and increases on either side of this temperature. Our results can be fitted to the simple expression

$$
\log _{10}\left(p_{e} \tau_{e c}\right)=A\left[\log _{10}\left(T_{e}\right)\right]^{2}+B \log _{10}\left(T_{e}\right)+C,
$$

TABLE II. Constants in the $e-V$ relaxation time $\tau_{e c}$ for 3000 $\mathrm{K} \leqslant T_{e} \leqslant 20000 \mathrm{~K}$.

\begin{tabular}{lccc}
\hline \hline \multicolumn{1}{c}{$T_{e}$ range $(\mathrm{K})$} & $A$ & $B$ & $C$ \\
\hline $3000-7000$ & 5.019 & -38.625 & 64.219 \\
$7000-20000$ & 2.448 & -18.704 & 25.635 \\
\hline
\end{tabular}

where $\tau_{e c}$ is in seconds, $p_{e}$ is in atmospheres, and the constants are given in Table II for $3000 \mathrm{~K} \leqslant T_{e} \leqslant 20000 \mathrm{~K}$. It is interesting to note that up to $T_{e}=20000 \mathrm{~K}$, the $w \neq 0 \rightarrow v>w$ transitions have a very small influence on the calculated value of $\tau_{e c}$. As a conclusion, when at $t=0 \mathrm{~s}$, all $\mathrm{N}_{2}$ molecules are populated in the ground state, the present study shows that for $3000 \mathrm{~K} \leqslant T_{e} \leqslant 20000 \mathrm{~K}$, the simple model proposed by Lee [Eqs. (1) and (8)] gives the right order of magnitude of the $e-V$ energy exchange term but underestimates it by an average factor of 3 .

\section{Influence of the anharmonicity of $\mathrm{N}_{2}$}

We carry out the same computations as in the preceding section in taking into account the anharmonicity of the $\mathrm{N}_{2}$ molecule. In Fig. 3, for $T_{e}=8000 \mathrm{~K}$, we note that the relaxation of low-lying levels is the same as in the harmonic case. On the contrary, the highly excited levels relax much more slowly in the anharmonic case. This results from the decrease of the $w \neq 0 \rightarrow v>w$ transition cross sections as $w$ increases. Then, even if in the anharmonic case, the energy gaps between high vibrational levels are smaller than in the harmonic case, the inefficiency of transitions between these vibrational levels results in a longer time to reach the final equilibrium. However, as the contribution to the vibrational energy is weighted toward the most populated vibrational levels, the influence of the anharmonicity of $\mathrm{N}_{2}$ on the vibrational energy relaxation will be significant only at high electron temperatures (when the high vibrational level populations become no longer negligible). For example, at $T_{e}=20000 \mathrm{~K}$, Fig. 4 shows the variations of the normalized vibrational energy relaxation rate

$$
\frac{d E_{v}^{n}}{d t}=\frac{d E_{v}}{d t} \frac{\tau_{e \text { Lee }}}{E_{v}^{*}}
$$

as a function of the normalized vibrational energy

$$
E_{v}^{n}=\frac{E_{v}}{E_{v}^{*}} .
$$

In this figure, we consider harmonic and anharmonic approximations but in both cases we use for $E_{v}^{*}$ the anharmonic equilibrium energy which is slightly higher than the harmonic equilibrium energy. Figure 4 confirms that deviations from the results obtained in the harmonic case are significant only near the final equilibrium, when the high vibrational level populations are no longer negligible. Then, the results obtained in the preceding section for a harmonic oscillator approximation can be used for $\mathrm{N}_{2}$ even for electron temperatures up to $20000 \mathrm{~K}$ as long as the system is far from the final Boltzmann equilibrium at $T_{e}$. We have also represented 


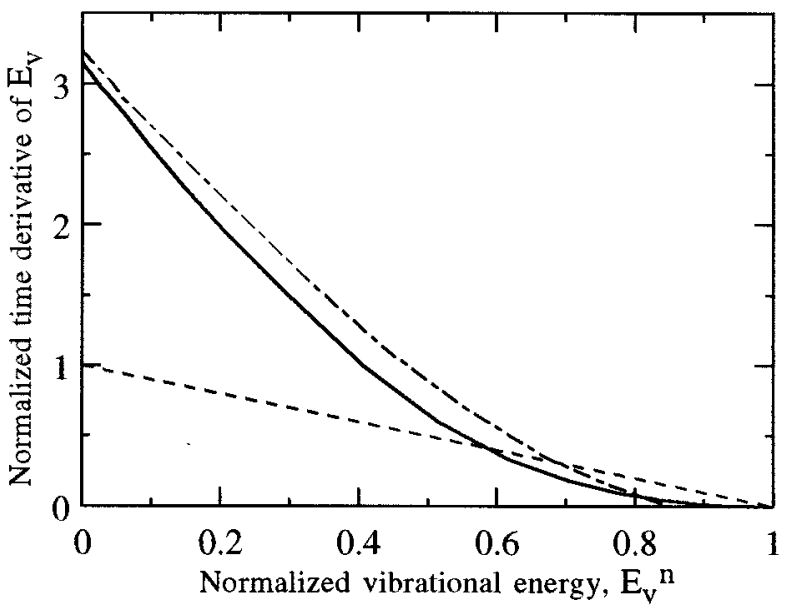

FIG. 4. For $T_{e}=20000 \mathrm{~K}$, the influence of the vibrational model of $\mathrm{N}_{2}$ on the variations of $d E_{v}^{n} / d t$ as a function of $E_{v}^{n}$. Dot-dashed line, harmonic oscillator approximation; solid line, anharmonic oscillator approximation; dashed line, Lee's model.

the curve obtained in using the expression proposed by Lee. This model appears to underestimate by a factor of 3 the $e-V$ energy transfer for short times and to overestimate it near the final equilibrium.

\section{INFLUENCE OF THE INITIAL VIBRATIONAL DISTRIBUTION}

In this section we propose to study the influence of an initial vibrational excitation of $\mathrm{N}_{2}$ on the $e-V$ energy exchange term for $3000 \mathrm{~K} \leqslant T_{e} \leqslant 20000 \mathrm{~K}$. In this paper we consider only situations where the vibrational distribution at $t=0 \mathrm{~s}$ is characterized by a vibrational temperature $T_{v}$. Two cases have to be considered, depending on the relative values of $T_{v}$ and $T_{e}$. In the following, we denote the case where $T_{e}>T_{v}$, an exciting condition, and the case where $T_{v}>T_{e}$, a relaxing condition. In exciting conditions with very low values of $T_{v}$, the population is mainly on the ground state at $t=0 \mathrm{~s}$ and then the situation is the same as the reference one considered in the preceding section. For plasma flows encountered in high enthalpy wind tunnels, an exciting condition corresponds to a shock around a blunt body $[9,11]$ and a relaxing condition corresponds to the expanding part of the plasma flow $[10,11]$ and to the boundary layer over a blunt body [12]. In order to make direct comparisons with the results obtained in the preceding section, we will show in detail the results obtained for the intermediate value $T_{e}=8000 \mathrm{~K}$.

\section{A. Exciting condition}

\section{1. $N_{2}$ is considered as a harmonic oscillator}

Figure 5 shows the time evolution of the vibrational level populations for $T_{e}=8000 \mathrm{~K}$ and $T_{v}=4000 \mathrm{~K}$ at $t=0 \mathrm{~s}$. As in the preceding sections, the low vibrational levels relax quicker than high ones. In this case, a time-dependent vibrational temperature appears to characterize the relaxation of low vibrational levels. In comparison with Fig. 3, we note that the initial vibrational distribution has a negligible influ-

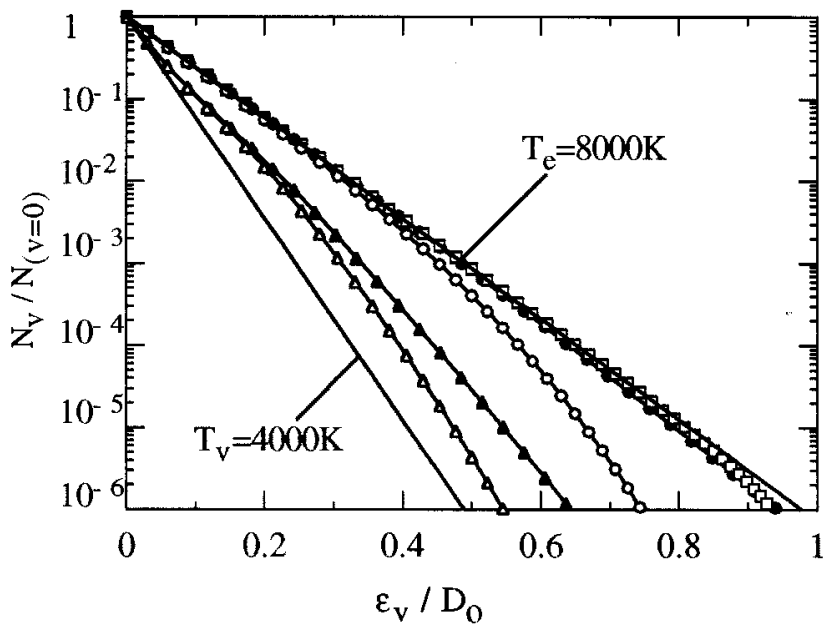

FIG. 5. Temporal relaxation of the vibrational level populations for $T_{e}=8000 \mathrm{~K}$ with, at $t=0 \mathrm{~s}, T_{v}=4000 \mathrm{~K} . N_{v}, \epsilon_{v}$, and the time $t$ are normalized as in Fig. 3. Closed symbols correspond to the harmonic oscillator approximation; open symbols correspond to the anharmonic oscillator approximation. Triangles, $t=10^{-1} \tau_{e \text { Lee }}$; circles, $t=\tau_{e \text { Lee }}$; squares, $t=10 \tau_{e \text { Lee }}$. Solid lines correspond to the initial and final Boltzmann distributions. The final equilibrium is reached at $t=10.5 \tau_{e \text { Lee }}$ in the harmonic case and at $t=3900 \tau_{e \text { Lee }}$ in the anharmonic case.

ence on the time necessary to reach the final equilibrium. For different electron temperatures, Fig. 6 shows that the value of $T_{v}$ at $t=0 \mathrm{~s}$ seems to have only a slight influence on the vibrational energy relaxation. This point is very interesting since it indicates that results obtained in the reference exciting condition (with at $t=0 \mathrm{~s}$, all $\mathrm{N}_{2}$ molecules populated in the ground state) can be used to describe accurately more general exciting conditions. Namely, for flow conditions where $3000 \mathrm{~K} \leqslant T_{e} \leqslant 20000 \mathrm{~K}$ and $T_{v} \leqslant T_{e}$, if $N_{2}$ is consid-

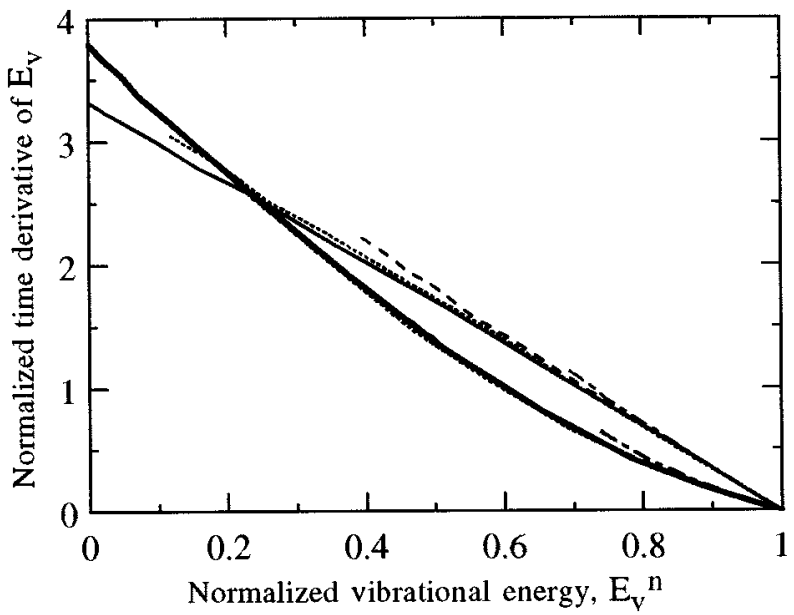

FIG. 6. With a harmonic oscillator approximation, the influence of the initial value of $T_{v}\left(<T_{e}\right)$ on the vibrational energy relaxation. Thin lines correspond to $T_{e}=8000 \mathrm{~K}$ and thick lines correspond to $T_{e}=20000 \mathrm{~K}$. Solid lines, at $t=0 \mathrm{~s}$, all $\mathrm{N}_{2}$ molecules are populated in the ground state; dotted lines, at $t=0 \mathrm{~s}, T_{v}=T_{e} / 4$; dashed lines, at $t=0 \mathrm{~s}, T_{v}=T_{e} / 2 ;$ dot-dashed lines, at $t=0 \mathrm{~s}$, $T_{v}=3 T_{e} / 4$. 


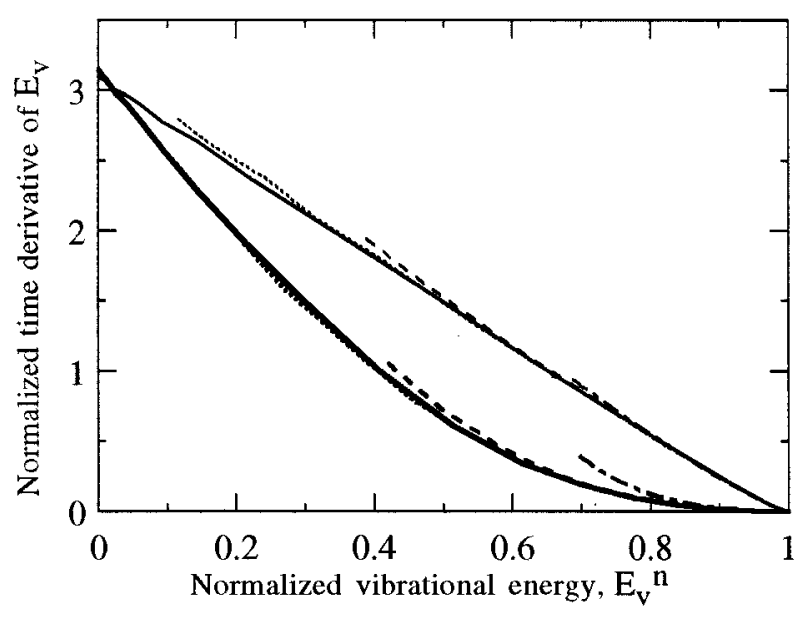

FIG. 7. With an anharmonic oscillator approximation, the influence of the initial value of $T_{v}\left(<T_{e}\right)$ on the vibrational energy relaxation. See Fig. 6 for notations.

ered as a harmonic oscillator, Eqs. (1) and (9) can be used to model accurately the $e-V$ energy exchange term.

\section{Influence of the anharmonicity of $\mathrm{N}_{2}$}

We carry out the same computations as in the preceding section in taking into account the anharmonicity of $\mathrm{N}_{2}$. In Fig. 5, as already observed, high vibrational levels relax more slowly in the anharmonic case than in the harmonic case. We note that an initial vibrational distribution increases significantly the time necessary to reach the final equilibrium in comparison with the reference condition (Fig. 3). For the same conditions as in Fig. 6, Fig. 7 represents the variations of $d E_{v}^{n} / d t$ as a function of $E_{v}^{n}$ with the anharmonic oscillator approximation. In this case too, the computed curves for the different vibrational temperatures remain very close to those obtained in considering at $t=0 \mathrm{~s}$ all $\mathrm{N}_{2}$ molecules populated in the ground state. The deviations observed when $T_{v}$ and $T_{e}$ are close to each other increase with temperature as the high vibrational level populations become no longer negligible. These deviations are the result of the slow relaxation of the high vibrational level populations. They point out the difficulty of determining accurately the $e-V$ energy exchange term near the final equilibrium. Finally, when $T_{v}<T_{e}$, we note that the results obtained with harmonic or anharmonic oscillator approximations remain close to each other. Then, conclusions drawn in Sec. III B 2 are also valid for a more general exciting condition and an anharmonic oscillator approximation.

\section{B. Relaxing condition}

When $T_{v}>T_{e}$, the vibrational energy at $t=0 \mathrm{~s}$ is higher than $E_{v}^{*}$, the vibrational energy corresponding to the equilibrium at $T_{e}$. Then during the simulation, $d E_{v} / d t$ is negative and increases with time up to zero at the final equilibrium.

\section{1. $\mathrm{N}_{2}$ is considered as a harmonic oscillator}

Figure 8 shows the time evolution of vibrational level populations for $T_{e}=8000 \mathrm{~K}$ and $T_{v}=20000 \mathrm{~K}$ at $t=0 \mathrm{~s}$. We

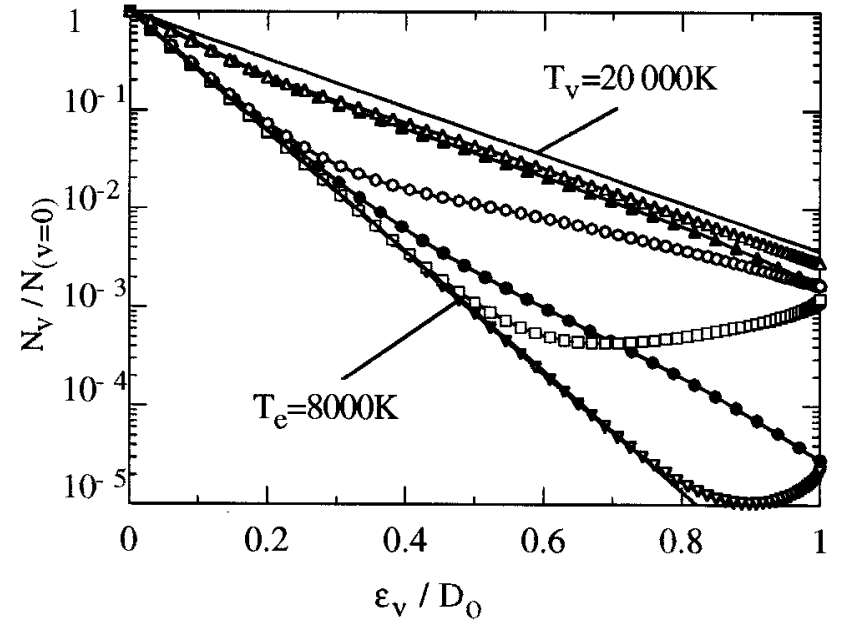

FIG. 8. Temporal relaxation of the vibrational level populations for $T_{e}=8000 \mathrm{~K}$ with, at $t=0 \mathrm{~s}, T_{v}=20000 \mathrm{~K} . N_{v}, \epsilon_{v}$, and the time $t$ are normalized as in Fig. 3. Closed symbols correspond to the harmonic oscillator approximation; open symbols correspond to the anharmonic oscillator approximation. Triangles up, $t=10^{-1} \tau_{e \text { Lee }}$; circles, $t=\tau_{e \text { Lee }} ;$ squares, $t=10 \tau_{e \text { Lee }} ;$ triangles down, $t$ $=10^{2} \tau_{e \text { Lee }}$. Solid lines correspond to the initial and final Boltzmann distributions. The final equilibrium is reached at $t=8.7 \tau_{e \text { Lee }}$ in the harmonic case and at $t=931 \tau_{e \text { Lee }}$ in the anharmonic case.

note that throughout the relaxation, the time evolution of low vibrational levels is characterized by a time-dependent vibrational temperature. As in the exciting condition, it is interesting to note that the initial vibrational distribution has a negligible influence on the time necessary to reach the final equilibrium. For $T_{e}=8000 \mathrm{~K}$ and different values of $T_{v}$ at $t=0 \mathrm{~s}$, Fig. 9 depicts the variations of $d E_{v}^{n} / d t$ as a function of $E_{v}^{n}$. First, this figure shows that the vibrational energy relaxation rate can be described by a linear rate equation.

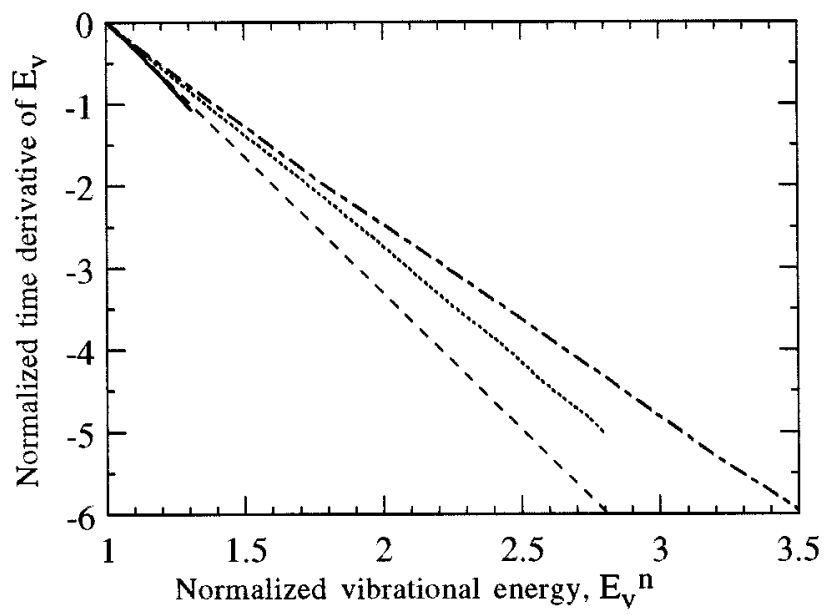

FIG. 9. With a harmonic oscillator approximation, the influence of the initial value of $T_{v}\left(>T_{e}\right)$ on the vibrational energy of relaxation at $T_{e}=8000 \mathrm{~K}$. Solid line, at $t=0 \mathrm{~s}, T_{v}=10000 \mathrm{~K}$; dotted line, at $t=0 \mathrm{~s}, T_{v}=20000 \mathrm{~K}$; dot-dashed lines, at $t=0 \mathrm{~s}$, $T_{v}=30000 \mathrm{~K}$. The dashed line represents the Landau-Teller relaxation of the vibrational energy calculated with the $e-V$ relaxation time $\tau_{e c}$ (see Fig. 1) corresponding to $T_{e}=8000 \mathrm{~K}$. 


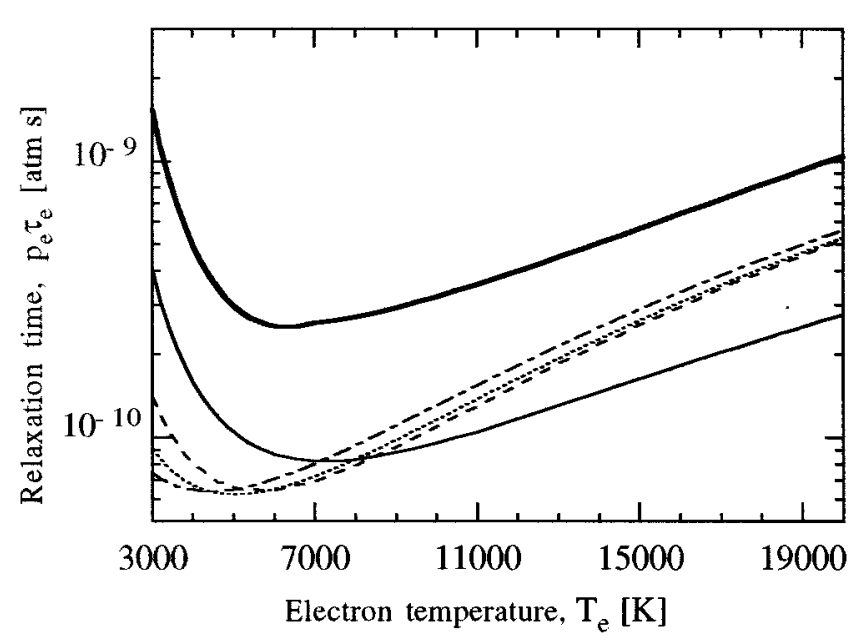

FIG. 10. With a harmonic oscillator approximation, the influence of the initial value of $T_{v}\left(>T_{e}\right)$ on the $e-V$ relaxation time. Thick solid line, $p_{e} \tau_{e \text { Lee }}$; thin solid line, $p_{e} \tau_{e c}$ (see Fig. 1); dashed line, at $t=0 \mathrm{~s}, T_{v}=T_{e}+2000 \mathrm{~K}$; dotted line, at $t=0 \mathrm{~s}$, $T_{v}=T_{e}+5000 \mathrm{~K}$; dot-dashed line, at $t=0 \mathrm{~s}, T_{v}=T_{e}+10000 \mathrm{~K}$.

Second it points out that the relaxation time $\tau_{e}$ (which is inversely proportional to the slope) depends on $T_{v}$. For 3000 $\mathrm{K} \leqslant T_{e} \leqslant 20000 \mathrm{~K}$, we have obtained the same characteristics for different values of $T_{v}-T_{e}$ and Fig. 10 presents the derived $e-V$ relaxation time. As the value of $T_{v}-T_{e}$ increases, we note that the minimum of the curve is translated towards lower electron temperatures. For $T_{e}<7000 \mathrm{~K}$, the $e-V$ relaxation time appears to be shorter in relaxing conditions than in exciting conditions. The discrepancy with the initial value proposed by Lee increases with $T_{v}-T_{e}$ and may exceed one order of magnitude. For $T_{e}>7000 \mathrm{~K}$, the $e-V$ relaxation time is shorter in exciting conditions than in relaxing conditions and the discrepancy with the initial value proposed by Lee decreases as $T_{v}-T_{e}$ increases. It is interesting to note that the computed $e-V$ relaxation time is nearly the same if the rate coefficients of the $w \neq 0 \rightarrow v>w$ transitions are assumed to be equal to those of the $0 \rightarrow v-w$ transitions [20].

\section{Influence of the anharmonicity of $\mathrm{N}_{2}$}

We carry out the same computations as in the preceding section in taking into account the anharmonicity of $\mathrm{N}_{2}$. In Fig. 8, as expected, we note that the relaxation of high vibrational levels is slower than in the harmonic case. It is interesting to note that the time necessary to reach the final equilibrium is nearly the same as the one corresponding to the reference exciting condition (Fig. 3) but is much shorter than the one corresponding to a more realistic exciting condition (Fig. 5). Near the final equilibrium, an inversion of population occurs due to the strong anharmonicity of $\mathrm{N}_{2}$. It is important to note that this inversion of population is theoretical since we have not considered any dissociation or recombination processes. However, as the populations of very high vibrational levels remain far smaller than those of low vibrational levels, this effect has no influence on the relaxation of the vibrational energy. Figure 11 is the same as Fig. 9 but in the anharmonic case. The higher the value of $T_{v}$, the more the vibrational energy relaxation rate deviates

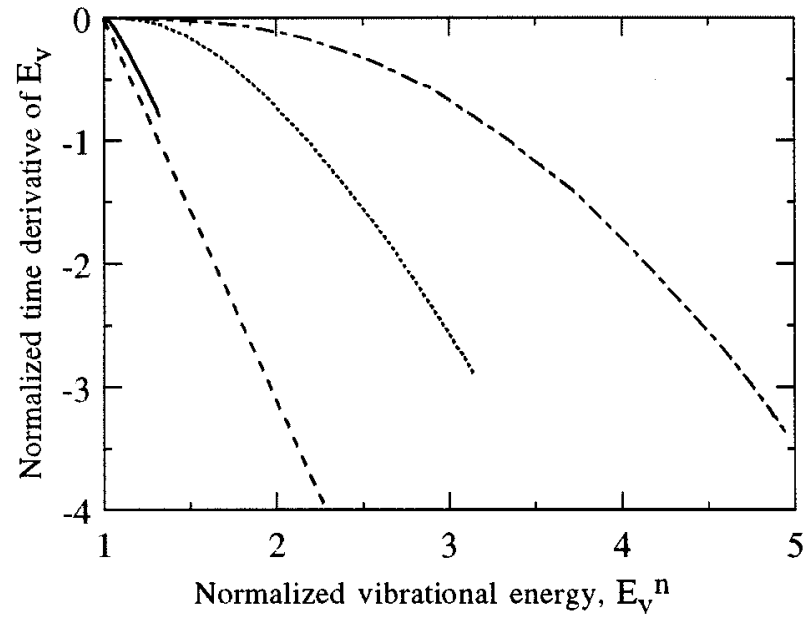

FIG. 11. With an anharmonic oscillator approximation, the influence of the initial value of $T_{v}\left(>T_{e}\right)$ on the vibrational energy relaxation at $T_{e}=8000 \mathrm{~K}$. See Fig. 9 for notations.

from a linear rate equation and the lower is the $e-V$ energy exchange term. It is important to note that we have obtained the same characteristics for different values of $T_{v}-T_{e}$. For relaxing conditions, this figure clearly points out that the simple linear model proposed by Lee is unadapted. Then, unfortunately, it appears impossible to extend to the anharmonic case the interesting results obtained in the preceding section for the harmonic case.

\section{CONCLUSION}

A numerical study of the $e-V$ energy exchange has been performed in nitrogen in order to examine the validity of the simple Landau-Teller-type model proposed by Lee. The accuracy of the present results depends on the set of $e-V$ cross sections. For $0 \rightarrow v>0$ transitions, the cross sections are now fairly well known. For the $w \neq 0 \rightarrow v>w$ transitions, the main data are theoretical and indicate that the cross sections decrease as $w$ increases. For the purpose of the present study, an approximate analytical expression has been used for all the $w \neq 0 \rightarrow v>w$ transition cross sections. To investigate the sensitivity of the results to the $e-V$ cross sections for $w \neq 0 \rightarrow v>w$ transitions, we have also carried out all the calculations of the present study in assuming that the rate coefficients of the $w \neq 0 \rightarrow v>w$ transitions are equal to those of the $0 \rightarrow v-w$ transitions [20]. In fact, the choice of the $e-V$ cross sections has appeared to influence only slightly the computed relaxation of the vibrational energy. Further theoretical and experimental work would be of great interest to establish an accurate set of $e-V$ cross sections for all transitions.

When $\mathrm{N}_{2}$ is considered as a harmonic oscillator, our computations have revealed that a linear Landau-Teller-type rate equation is accurate to describe the relaxation rate of vibrational energy for $3000 \mathrm{~K} \leqslant T_{e} \leqslant 20000 \mathrm{~K}$. For the theoretical initial situation studied by Lee (at $t=0 \mathrm{~s}$, all $\mathrm{N}_{2}$ molecules are populated in the ground state) we have shown that the characteristic relaxation time proposed by this author turned out to be overestimated by an average factor of 3 . In this case, we propose an analytical expression of the $e-V$ relax- 
ation time [Eq. (9)]. In order to consider general flow conditions, we have studied the influence of the vibrational distribution on the $Q_{e-V}$ term modeling for $3000 \mathrm{~K} \leqslant T_{e} \leqslant 20000$ $\mathrm{K}$. We have considered only situations where the initial vibrational distribution is characterized by a vibrational temperature $T_{v}$. It is found that results obtained, in considering at $t=0 \mathrm{~s}$, all $\mathrm{N}_{2}$ molecules populated in the ground state are accurate to describe exciting conditions $\left(T_{v}<T_{e}\right)$. In relaxing conditions $\left(T_{v}>T_{e}\right)$, we have observed that the $e-V$ relaxation time depends on the initial vibrational temperature. For $T_{e}<7000 \mathrm{~K}$, the $e-V$ relaxation time appears to be shorter in relaxing conditions than in exciting conditions and the discrepancy increases as $T_{e}$ decreases. For $T_{e}>7000 \mathrm{~K}$, the $e-V$ relaxation time appears to be shorter in exciting conditions than in relaxing conditions and the discrepancy increases with $T_{e}$.

When the anharmonicity of $\mathrm{N}_{2}$ is taken into account we have pointed out the difficulty of modeling the $Q_{e-V}$ term near the final equilibrium at the electron temperature $T_{e}$. In exciting conditions and for $3000 \mathrm{~K} \leqslant T_{e} \leqslant 20000 \mathrm{~K}$, we have observed that results obtained in the harmonic case are accurate enough to times near the initial condition of the simulation. For relaxing conditions and for $3000 \mathrm{~K} \leqslant T_{e} \leqslant 20000 \mathrm{~K}$, the relaxation of vibrational energy generally does not follow a Landau-Teller-type rate equation. Then it appears much more difficult to model accurately the $Q_{e-V}$ term in relaxing conditions than in exciting conditions. Furthermore, the present study of relaxing conditions has been limited to conditions where a time-dependent vibrational temperature characterizes the low vibrational level distribution. In more general cases, larger deviations from a linear Landau-Teller-type rate equation may be expected.

However, it is interesting to note that the present study has confirmed the order of magnitude of the $e-V$ energy exchange term obtained by Lee and then the very high efficiency of the $e-V$ coupling in nitrogen. Hence in partially ionized nitrogen flows (where the electron number density is high enough), it validates the existence of the local equilibrium $T_{v}=T_{e}$. However, in weakly ionized nitrogen flows, the present study has clearly put forward that the very simple model proposed by Lee should be implemented with care in flow codes and might either overestimate or underestimate the $e-V$ energy exchange term.

Finally, the present results have to be considered as a first step towards a model for the $e-V$ coupling that will represent a balance between the ease of implementation in a computational fluid dynamics method and an accurate description of the $e-V$ coupling physics.
[1] G. V. Candler, in Proceedings of the Second European Symposium on Aerothermodynamics for Space Vehicles, ESA SP367, edited by J. J. Hunt (European Space Agency, Noordwijk, 1995), p. 365.

[2] C. Park, in Molecular Physics and Hypersonic Flows, edited by M. Capitelli (Kluwer, Dordrecht, 1996), Vol. C482, p. 581.

[3] L. Landau and E. Teller, Phys. Z. Sowjetunion 10, 34 (1936).

[4] C. Park, J. Thermophys. Heat Transfer 3, 233 (1989).

[5] J. Olejniczak and G. V. Candler, Phys. Fluids 7, 1764 (1995).

[6] J. H. Lee, in Thermal Design of Aeroassisted Orbital Transfer Vehicles, edited by H. F. Nelson (AIAA, New York, 1985), Vol. 96, p. 3.

[7] J. H. Lee, in Thermophysical Aspects of Re-entry Flows, edited by J. N. Moss and C. D. Scott (AIAA, New York, 1986), Vol. 103, p. 197.

[8] J. Keck and G. Carrier, J. Chem. Phys. 43, 2284 (1965).

[9] G. V. Candler, Ph.D. thesis, Standford University, 1988 (unpublished).

[10] P. Domingo, A. Bourdon, and P. Vervisch, Phys. Plasmas 2, 2853 (1995).

[11] C. Park, Non-Equilibrium Hypersonic Aerothermodynamics (Wiley, New York, 1990).
[12] A. Bourdon and P. Vervisch (unpublished).

[13] I. K. Dmitrieva and V. A. Zenevich, Chem. Phys. Lett. 96, 228 (1983).

[14] I. K. Dmitrieva, S. K. Pogrebnya, and P. I. Porshnev, Chem. Phys. 142, 25 (1990).

[15] G. J. Schulz, in Principles of Laser Plasmas, edited by G. Bekefi (Wiley, New York, 1976), p. 33.

[16] W. M. Huo, V. McKoy, M. A. P. Lima, and T. L. Gibson, in Thermophysical Aspects of Re-entry Flows (Ref. [7]), Vol. 103 , p. 152.

[17] L. A. Morgan, J. Phys. B 19, L439 (1986).

[18] M. Allan, J. Phys. B 18, 4511 (1985).

[19] M. Allan (private communication).

[20] M. Capitelli and M. Dilonardo, Rev. Phys. Appl. 13, 115 (1978).

[21] L. Dubé and A. Herzenberg, Phys. Rev. A 20, 194 (1979).

[22] B. F. Gordiets, C. M. Ferreira, V. L. Guerra, J. M. A. H. Loureiro, J. Nahorny, D. Pagnon, M. Touzeau, and M. Vialle, IEEE Trans. Plasma Sci. 23, 750 (1995).

[23] A. C. Hindmarsh, ACM SIGNUM Newslett. 15 (1980).

[24] S. Slinker and A. W. Ali, Naval Research Laboratory Memorandum Report No. 4756, 1982 (unpublished). 